\title{
Important Role of Mitochondria and the Effect of Mood Stabilizers on Mitochondrial Function
}

\author{
M. LUPTÁK ${ }^{1}$, J. HROUDOVÁ ${ }^{1,2}$ \\ ${ }^{1}$ Department of Pharmacology, First Faculty of Medicine, Charles University and General \\ University Hospital in Prague, Prague, Czech Republic, ${ }^{2}$ Department of Psychiatry, First Faculty of \\ Medicine, Charles University and General University Hospital in Prague, Prague, Czech Republic
}

Received August 13, 2019

Accepted August 27, 2019

\begin{abstract}
Summary
Mitochondria primarily serve as source of cellular energy through the Krebs cycle and $\beta$-oxidation to generate substrates for oxidative phosphorylation. Redox reactions are used to transfer electrons through a gradient to their final acceptor, oxygen, and to pump hydrogen protons into the intermembrane space. Then, ATP synthase uses the electrochemical gradient to generate adenosine triphosphate (ATP). During these processes, reactive oxygen species (ROS) are generated. ROS are highly reactive molecules with important physiological functions in cellular signaling. Mitochondria play a crucial role in intracellular calcium homeostasis and serve as transient calcium stores. High levels of both, ROS and free cytosolic calcium, can damage mitochondrial and cellular structures and trigger apoptosis. Impaired mitochondrial function has been described in many psychiatric diseases, including mood disorders, in terms of lowered mitochondrial membrane potential, suppressed ATP formation, imbalanced $\mathrm{Ca}^{2+}$ levels and increased ROS levels. In vitro models have indicated that mood stabilizers affect mitochondrial respiratory chain complexes, ROS production, ATP formation, $\mathrm{Ca}^{2+}$ buffering and the antioxidant system. Most studies support the hypothesis that mitochondrial dysfunction is a primary feature of mood disorders. The precise mechanism of action of mood stabilizers remains unknown, but new mitochondrial targets have been proposed for use as mood stabilizers and mitochondrial biomarkers in the evaluation of therapy effectiveness.
\end{abstract}

\section{Key words}

Mitochondria - Oxidative phosphorylation - Reactive oxygen species $\bullet$ Calcium • Mitochondrial signaling • Mood stabilizers

\section{Corresponding author}

M. L'upták, Department of Pharmacology, First Faculty of Medicine, Charles University and General University Hospital in Prague, Albertov 4, 12800 Prague 2, Czech Republic. E-mail: mat.luptak@gmail.com

\section{Introduction}

\section{Mitochondria as cellular organelles}

The mitochondrion is one of the largest organelles in human cells, occupying approximately $25 \%$ of the cytoplasmic volume. Evolutionarily, they are presumed to be descendants of bacteria on the basis of the endosymbiotic hypothesis of mitochondria origin. This hypothesis may explain the origin of the two organelle membranes border the two mitochondrial compartments: the intermembrane space and the matrix. The outer mitochondrial membrane is smooth and permeable to solutes smaller than $5 \mathrm{kDa}$. The inner mitochondrial membrane (IMM) is mostly impermeable and contains many transport channels. Its surface is enlarged by cristae, with a higher concentration of integral proteins, which enable adenosine triphosphate (ATP) formation (Lodish et al. 2013, Srivastava et al. 2018). In addition to ATP production, mitochondria have a role in $\mathrm{Ca}^{2+}$ homeostasis, production and disposal of reactive oxygen species (ROS), apoptosis regulation and thermoregulation (de Sousa et al. 2014).

Mitochondria are highly dynamic organelles that undergo frequent fusion (merging) and fission (dividing). Fission and fusion were likely developed to prevent the 
accumulation of mutated mitochondrial DNA. They are optimal tools for the isolating of damaged segments. Disrupted mitochondrial dynamics are often linked to genetic disorders, indicating that mitochondrial dynamics play important roles in mitochondrial physiology (Lodish et al. 2013).

\section{Mitochondria as energy factories}

The primary function of mitochondria is to use products of glycolysis, proteolysis, or lipolysis and oxygen through a series of a biochemical reactions leading to ATP formation (Andreazza and Nierenberg 2018). ATP is created via oxidative phosphorylation (OXPHOS) using the electron transport chain (ETC) as the machinery (Clay et al. 2011).

The pyruvate dehydrogenase complex, which converts pyruvate into acetyl-coenzyme A (acetyl-CoA), is able to control carbon entry from two main sources: carbohydrates and glucogenic amino acids. Fatty acids are metabolized in another way; first, they are converted into acyl-coenzyme A, attached to carnitine, and shuttled into the mitochondria. Second, acyl-CoA undergoes $\beta$-oxidation and results in the production of acetyl-CoA. Acetyl group from acetyl-CoA is oxidized into $\mathrm{CO}_{2}$ concurrently with the production of substrates for OXPHOS: the reduced forms of high-energy carriers nicotinamide adenine dinucleotide (NADH) and flavin adenine dinucleotide $\left(\mathrm{FADH}_{2}\right)$. The presence of oxygen is necessary to regenerate the oxidized forms of the electron carriers nicotinamide adenine dinucleotide $\left(\mathrm{NAD}^{+}\right)$and flavin adenine dinucleotide $\left(\mathrm{FAD}^{+}\right)$(Lodish et al. 2013, de Sousa et al. 2014).

Mitochondria function through a highly effective transport gradient in which electrons move from acceptors with less electronegativity to those with greater electronegativity at the same time that the redox reactions involving both, electron donors and acceptors are in progress. Simultaneously, through electron transport, $\mathrm{H}^{+}$ions are pumped into the intermembrane space (IMS), increasing the $\mathrm{pH}$ in the matrix, which increases its electronegativity, thereby creating proton motive force (PMF) (Divakaruni and Brand 2011, Lodish et al. 2013).

The ETC consists of 4 complexes with supramolecular organization, all of which have several prosthetic groups as heme or iron-sulfur clusters. Hemebased prosthetic groups are bound to cytochromes and are able to delocalize accepted electrons due to their porphyrin structure. Every cytochrome $c$ (cyt $c$ ) molecule has a different reduction potential, and the electrons flow downhill. Iron-sulfur clusters disperse accepted electrons among iron atoms, and their true charge is approximately $2^{+}-3^{+}$. Complexes I, III and IV are responsible for pumping $\mathrm{H}^{+}$into the IMS (Lodish et al. 2013, Ohnishi et al. 2018).

Complex I, NADH-CoQ oxidoreductase, is a large, multi-subunit enzyme with a noncovalently bound flavin mononucleotide (Ohnishi et al. 2018). Complex I serves as an entry point into the ETC and a key component for the respiration. The high-energy carrier NADH transfers two electrons to complex I, which catalyzes the regeneration of the reduced NADH and electrons are transferred to flavin mononucleotides and, then, further through the iron-sulfur cluster to oxidized coenzyme Q10 (CoQ10), also known as ubiquinone, which is reduced to ubiquinol (Hirst 2009). These redox reactions are exergonic, and every transported electron loses some potential in the process. The released energy is used for actively pumping four $\mathrm{H}^{+}$ across the IMM (Fig. 1) (Lodish et al. 2013, Ohnishi et al. 2018). This reaction is completely reversible, and through PMF, the electrons can be transferred backward, while a massive amount of superoxide anion is produced in a process known as reverse electron transfer (Dubouchaud et al. 2018). Complex I is easily inhibited by psychopharmaca; therefore, it is an interesting target for further research (Hroudova and Fisar 2010).

$\mathrm{NADH}+\mathrm{CoQ}+6 \mathrm{H}^{+}{ }_{\text {in }} \rightarrow \mathrm{NAD}^{+}+\mathrm{H}^{+}{ }_{\text {in }}+\mathrm{CoQH}_{2}+4 \mathrm{H}^{+}$out

Complex II, succinate dehydrogenase, is a four subunit lipoprotein with a large soluble domain and a smaller integral-membrane domain (Hroudová and Fišar 2013, Iverson 2013). It is the smallest respiratory chain complex and is fully encoded by nuclear DNA (Grimm 2013). Complex II partly consists of covalently bound flavin adenine dinucleotide, several redox active centers, iron-sulfur clusters and quinone binding sites (Cecchini 2013). Succinate dehydrogenase enables the side entry of high energy carriers into the ECT, specifically reduced $\mathrm{FADH}_{2}$, which is formed in the Krebs cycle during the oxidation of succinate to fumarate, which occurs in the soluble domain of complex II (Grimm 2013, Iverson 2013). Complex II has a role in OXPHOS by participating in the reversible oxidoreduction of the quinol and quinone (Grimm 2013). Succinate dehydrogenase is directly involved in the Krebs cycle: two electrons are released during succinatefumarate oxidation are transferred via $\mathrm{NAD}^{+}$through 
iron-sulfur cluster to CoQ10 (ubiquinone), reducing it to ubiquinol (Fig. 1) (Lodish et al. 2013).

$$
\mathrm{Succ}+\mathrm{CoQ} \rightarrow \mathrm{Fum}+\mathrm{CoQH}_{2}
$$

First, a partially reduced semiquinone radical is formed, which is immediately stabilized after the second electron is transferred. This mechanism most likely serves prevents excessive electron leakage (Grimm 2013). All reactions catalyzed via succinate dehydrogenase are reversible, although they are more efficient in their preferred catalytic direction. However, this enzyme reversibility may link complex II with some metabolic diseases (Cecchini 2013). CoQ10 is the main transporter of electrons from complex I and II to complex III (Rodriguez-Hernandez et al. 2009). Energy released during this step is not sufficient to pump $\mathrm{H}^{+}$into the IMS because part of the energy is absorbed during the reduction of CoQ10; thus, the PMF is not generated by this reaction (Lodish et al. 2013). Recent studies have suggested that the subunits of complex II might have tumor-suppressor gene function. Complex II can be then involved in apoptosis, thereby acting as a pro-apoptotic sensor and transducer of apoptotic signals (Grimm 2013).

Complex III, CoQ : cyt $c$ oxidoreductase, is a transmembrane enzyme complex consists of two centers, $\mathrm{Q}_{\mathrm{i}}$ facing the matrix and $\mathrm{Q}_{\mathrm{o}}$ oriented toward to IMS, and three main subunits: cyt $c_{1}$, the Rieske center and cyt $b$ (Iwata et al. 1998, Hroudová and Fišar 2013). Complex III catalyzes the oxidation of one molecule of reduced coenzyme $\mathrm{Q}\left(\mathrm{CoQH}_{2}\right)$ and the reduction of two molecules of cyt $c$. It pumps $4 \mathrm{H}^{+}$into the IMS and absorbs $2 \mathrm{H}^{+}$from the matrix in a two-step process called the Q cycle (Speijer 2018).

$$
\mathrm{CoQH}_{2}+2 \mathrm{cyt} c^{3+}+2 \mathrm{H}^{+} \rightarrow \mathrm{CoQ}+4 \mathrm{H}^{+}+2 \mathrm{cyt} c^{2+}
$$

One molecule of $\mathrm{CoQH}_{2}$ is oxidized at the $\mathrm{Q}_{\mathrm{o}}$ center, two protons are released into the IMS, two electrons are released and CoQ dissociates from complex III. However, each cyt $c$ is can carry only one electron, which is the reason that the $\mathrm{Q}$ cycle is a two-step reaction. The first electron travels via the high-potential chain through the Rieske center (iron-sulfur clusters) and cyt $c_{l}$ and finally reduces cyt $c$. The second electron travels via a low-potential chain ( $\mathrm{Q}_{\mathrm{i}}$ center) through cyt $b_{L}$ and $\mathrm{b}_{\mathrm{H}}$ to partially reduce CoQ and create a stable semiquinone radical. The second step of the Q cycle is exactly the same as that of a new $\mathrm{CoQH}_{2}$ binding to complex III, and the second electron completes the reduction of semiquinone. Complex III consumes $2 \mathrm{H}^{+}$protons from the matrix, and the reduced $\mathrm{CoQH}_{2}$ is recycled (Fig. 1). Altogether, a normally operating complex III both produces and consumes $\mathrm{CoQH}_{2}$ (Lodish et al. 2013, Speijer 2018).

Complex IV, cytochrome $c$ oxidase, consists of 13 subunits, of which only three form the catalytic core of the complex. Complex IV enables the terminal reduction of oxygen to $\mathrm{H}_{2} \mathrm{O}$ (Hroudová and Fišar 2013, Lodish et al. 2013).

$$
4 \text { cyt } c^{2+}+8 \mathrm{H}^{+}{ }_{\text {in }}+\mathrm{O}_{2} \rightarrow 4 \text { cyt } c^{3+}+2 \mathrm{H}_{2} \mathrm{O}+4 \mathrm{H}^{+}{ }_{\text {out }}
$$

Reduction proceeds partially when 4 molecules of reduced cyt $c$ bind sequentially to complex IV and transfer the electron from its heme first to the $\mathrm{Cu}_{\mathrm{a}}{ }^{2+}$ ions, then to the heme in cyt $a$, next to the $\mathrm{Cu}_{\mathrm{b}}{ }^{2+}$ ions and finally to the heme of cyt $a_{3}$. All together, these results create the oxygen reduction center, creating four molecules of $\mathrm{H}_{2} \mathrm{O}$ (Fig. 1). During this process, another four $\mathrm{H}^{+}$are translocated ( 2 per every $1 / 2$ of $\mathrm{O}_{2}$ ) across the membrane (Lodish et al. 2013). The important regulator of this complex is the protein kinase $\mathrm{A}$ /cyclic adenosine monophosphate (PKA/cAMP) signaling pathway, which stimulates the phosphorylation of subunit IV and increases the activity of complex IV (Bouchez and Devin 2019).

Complex V, ATP synthase, is the fifth and the last enzyme of the OXPHOS process and contains two functional domains: $F_{O}$ and $F_{1} . F_{O}$ is a domain with three types of integral membrane proteins $-a, b$ and $c$. Protein $a$ serves as an ion channel and is rigidly connected to $b$ and protein $c$ forms a " $c$ ring". $F_{1}$ is a hydrophilic catalytic domain containing five polypeptides with the composition $\alpha_{3} \beta_{3} \gamma \delta \varepsilon$ that are strongly bound to the $\mathrm{F}_{\mathrm{O}}$ at the surface of the membrane. $F_{1}$ contains the catalytic machinery for both, ATP synthesis and hydrolysis. The domains are connected via two stalks: subunit $\gamma$ of the $F_{1}$, which is firmly bound to the rotating cavity of the $F_{O}$ " $c$ ring", and subunit $b$ of $\mathrm{F}_{\mathrm{O}}$, which serves as a stator and prevents the $\alpha_{3} \beta_{3}$ hexamer situated on the top of subunit $\gamma$ from rotating (Fig. 1) (Jonckheere et al. 2011, Lodish et al. 2013, Neupane et al. 2019). Complex V exists as both a monomer and a dimer supercomplex, which optimizes cristae shape and proton flow (Barca et al. 2018). ATP synthase is the smallest biological nanomotor and is responsible for ATP creation, which it generates using a proton gradient with membrane potential as a battery. 
This battery is powered by decreased $\mathrm{pH}$ and a higher concentration of $\mathrm{H}^{+}$ions in the IMS and increased $\mathrm{pH}$ and a lower concentration of $\mathrm{H}^{+}$ions in the mitochondrial matrix. This situation is a result of $\mathrm{H}^{+}$pumping across the IMM during electron movement through the ETC. The electrochemical energy that is thus generated is translated to the PMF: $\mathrm{H}^{+}$ions return to the matrix through the $a$ subunit of the $\mathrm{F}_{\mathrm{O}}$ domain and power ATP synthesis. The binding-change mechanism is describes the rotation mechanism of ATP synthase during the synthesis of ATP (Neupane et al. 2019). All three $\beta$ subunits are able to bind adenosine diphosphate and phosphate to create ATP during indirect coupling with the proton flow of the exoplasmic medium. The PMF is used for the rotation of the " $c$ ring" with subunit $\gamma$ to induce cyclical changes in the $\beta$ subunits during the $360^{\circ}$ turnover. There are three conformational states of subunit $\beta$ : open, loose and tight, all of which have a different affinity for substrates and products. At least two protons must past through the membrane for synthesis of one molecule of ATP. Synthesis and hydrolysis of ATP depend only on the direction of the rotation (Lodish et al. 2013, Neupane et al. 2019). When the IMM is not sufficiently polarized, complex $\mathrm{V}$ acts as an ATP hydrolase and uses released energy from the hydrolyzed ATP to move the $\mathrm{H}^{+}$protons uphill and restore the $\Delta \mathrm{p}$ (Nesci et al. 2016). However, Liu et al. (2016) disagree with the conventional model that indicates reliance on the rotating " $c$ ring". In their opinion, the model is defective because the number of c subunits $(8-15)$ is indivisible by the number of rotations $\left(3 \times 120^{\circ}=360^{\circ}\right)$. They propose an alternative rotary model, inspired by the mechanism of a retractable click ballpoint pen, in which the $\alpha_{3} \beta_{3}$ hexamer turns instead of the "c ring" (Liu et al. 2016). Complex V is able to undergo dimerization and oligomerization, which facilitates ATP synthesis and is beneficial for the cell (Jonckheere et al. 2012).

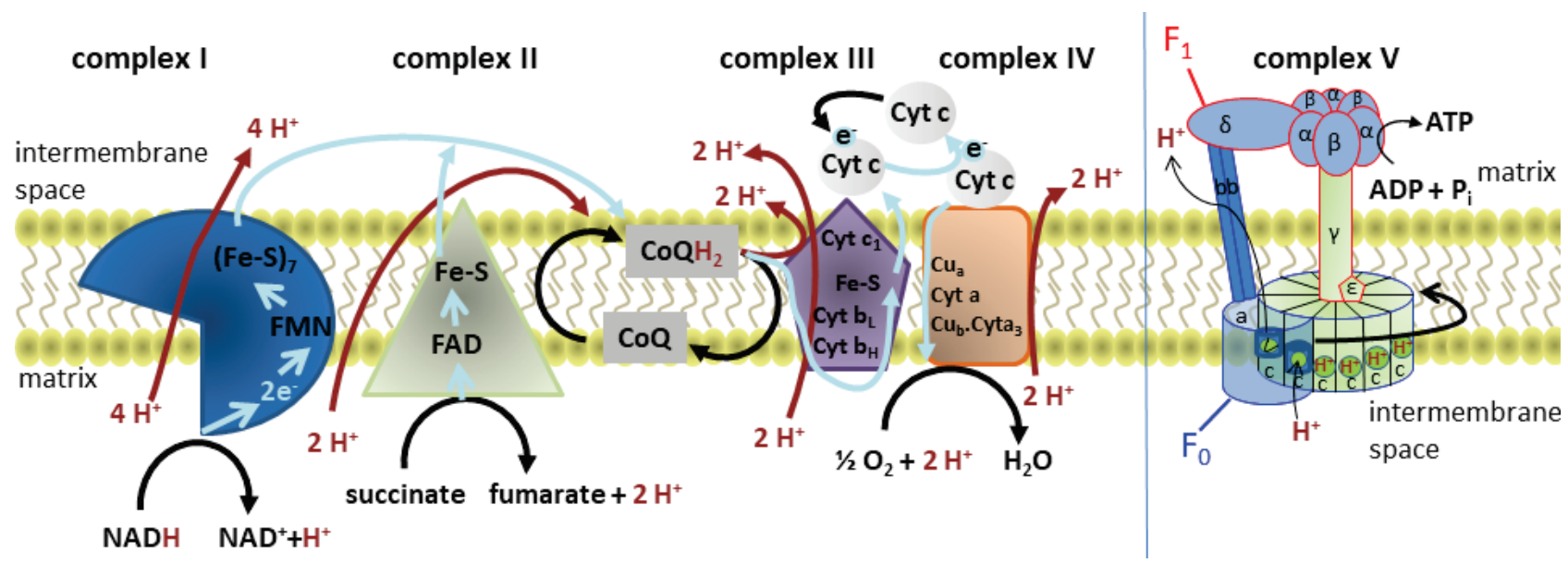

Fig. 1. The electron transport chain and structure of ATP synthase (adapted from Lodish et al. 2013). Electrons (blue arrows) from reduced nicotine adenine dinucleotide (NADH) move through complex I via flavin mononucleotide (FMN) and iron-sulfur clusters (Fe-S) and together with 2 protons, they bind oxidized coenzyme Q (CoQ) to form reduced coenzyme Q $\left(\mathrm{CoQH}_{2}\right)$. The electron flow enables $4 \mathrm{H}^{+}$(red arrows) to be pumped into the intermembrane space. Complex II enables electron side entry and the electrons move from succinate through oxidized flavin adenine dinucleotide $\left(\mathrm{FAD}^{+}\right)$and $\mathrm{Fe}-\mathrm{S}$ form $\mathrm{CoQH}_{2}$. Electrons flow from $\mathrm{CoQH} \mathrm{H}_{2}$ to complex III and then via cytochrome $c\left(\right.$ Cyt $c$ ) to complex IV. In total, $10 \mathrm{H}^{+}$per one NADH or $6 \mathrm{H}^{+}$per one FADH $\mathrm{F}_{2}$ are translocated into the intermembrane space. Complex $\mathbf{V}$ is formed from the stator (blue) and rotor (green). The $F_{0}$ domain of ATP synthase consists of $3 \mathbf{a}$ and $3 \mathbf{b}$ subunits, and $10 \mathbf{c}$ subunits, which form the c-ring. Subunit a contains the $\mathrm{H}^{+}$ion half-channel the mediates proton movement across the membrane. The subunits $\mathbf{a}$ and $\boldsymbol{\beta}$ of $F_{1}$ form a hexamer on the top of the $\mathbf{y}$ subunit that is inserted into the c-ring.

\section{Mitochondrial ROS production}

ROS are highly unstable molecules which can damage different cellular structures, e.g. lipids, proteins and nucleic acids. The most common forms of ROS are superoxide anion, hydrogen peroxide and hydroxyl radical. The physiological concentration of ROS is important for maintaining homeostasis and transducing signals, whereas massive ROS production is connected with the induction of apoptosis, necrotic cell death, and pathophysiological consequences (Lanciano et al. 2013).

In mitochondria, ROS are byproducts of OXPHOS, which couple substrate with adenosine diphosphate phosphorylation. This coupling is not complete because some protons are able to return to the matrix via other pathways that enable them to bypass ATP synthase. This process is known as a basal proton 
leak (Jastroch et al. 2010). Electron leakage occurs when electrons avoid some part of the ETC and partially reduce oxygen to create superoxide, which is superoxide anion in aqueous environments. Superoxide anion may be converted to hydrogen peroxide, catalyzed by superoxide dismutase, which is transformed into the most damaging product: hydroxyl radical (Jastroch et al. 2010, Lanciano et al. 2013). In OXPHOS superoxide is generated mostly by complex I and complex III. Complex I releases it into the matrix, and complex III releases it on both sides of the IMM (Hroudová and Fišar 2013). There are several ways in which ROS escape out of the mitochondrial matrix. Hydrogen peroxide is able to cross phospholipid barriers, but membrane pores such as mitochondrial permeability transition pore (mPTP), aquaporins and anion channels play crucial roles (Daiber et al. 2017).

There are also other sources of mitochondrial ROS, such as monoamine oxidase, catabolizes monoamines and produce hydrogen peroxide. Another mitochondrial enzyme, $\alpha$-ketoglutarate dehydrogenase, produces $\mathrm{H}_{2} \mathrm{O}_{2}$, especially when the ratio of NADH and $\mathrm{NAD}^{+}$is high (Hroudová and Fišar 2013). Mitochondria can also stimulate neighboring mitochondria to release more ROS in a process called ROS-induced ROS release. ROS are able to activate inner membrane anion channels, the $\mathrm{mPTP}$, and ATP-sensitive $\mathrm{K}^{+}$channels to disrupt mitochondrial membrane potential. High amounts of $\mathrm{NADH}$ and $\mathrm{FADH}_{2}$ must be consumed to compensate for the activation of these channels, which causes even greater production of ROS (Bertero and Maack 2018). Interestingly, the production of ROS is lower when complex I and complex III assemble to form a supercomplex (Bolanos et al. 2016).

Under physiological conditions, ROS are neutralized by and antioxidant system consisting of different enzymes, such as superoxide dismutase, catalase, glutathione peroxidase and glutathione transferase. The superoxide is converted to hydrogen peroxide by superoxide dismutase and is then eliminated by glutathione peroxidase or peroxiredoxin. This system, together with other antioxidant molecules, such as coenzyme Q10, creatine and tocopherol, protects tissues from oxidative damage, but its efficiency might be decreased under pathophysiological conditions (Wang 2007, de Sousa et al. 2014, Bertero and Maack 2018).

Increased production of ROS occurs when the membrane potential is low, mitochondria are depolarized or a response is mounted based on hypoxia or calcium signaling (Angelova and Abramov 2018).
Overproduction of ROS or dysregulation in the antioxidant system damages lipids, proteins and nucleic acids and the structures of which they are part (Bolisetty and Jaimes 2013). Prolonged overproduction causes oxidative stress and induce cell death, which is associated with many pathologies, including neurodegeneration, obesity or diabetes (Mailloux and Harper 2012). Mitochondrial permeability is largely increased under inflammatory conditions, which enables even more ROS to escape to the cytosol (Daiber et al. 2017). Complex I generates a significant amount of superoxide anions during reverse electron transfer, during which electrons are transferred backwards, from quinone to $\mathrm{NAD}^{+}$ (Dubouchaud et al. 2018). These superoxides are especially likely to be produced under hypoxic conditions. During hypoxia, electron flow stops, and the mitochondrial membrane potential drops. ATP is then taken up by mitochondria from the cytosol and hydrolyzed by ATP synthase, and the mitochondrial membrane potential is restored. However, the impaired electron flow leads to only partial oxygen reduction and contributes to increased ROS production (Bernardi et al. 2015). Complex II is considered to be an important regulator of the ROS production by complexes I and III and may be a major source of ROS only during heart failure (De Giusti et al. 2013).

The mitochondrial dysfunction and the increased ROS production are probably one of the main factors in brain aging processes. During the aging, the number of defective mitochondria that produce more ROS is increased and the antioxidant system is also less effective. Decreased OXPHOS capacity and mitochondrial DNA damage is also described (Hroudová and Fišar 2013, Cedikova et al. 2016). Because the brain is the greatest oxygen consumer in the body, brain cells are the most vulnerable to oxidative damage (Wang 2007). In addition, ROS and reactive nitrogen species are also considered key factors in cardiovascular and inflammatory diseases. Superoxide acts as a direct antagonist of nitric oxide, which is endogenous vasodilator. Their reaction produces peroxynitrite which leads to endothelial dysfunction of vessels (Daiber et al. 2017).

\section{Mitochondrial ROS and signaling}

The initial studies conducted in 1998 revealed that mitochondria release hydrogen peroxide under hypoxic conditions. Increased levels of hydrogen peroxide activate hypoxia-inducible factor, which is important transcription factor for the metabolic 
adaptation to low oxygen levels (Chandel et al. 1998). Currently, ROS are considered the secondary signaling messengers; that is, they change the redox state of the signal transduction proteins (Lenaz 2012). The signaling tasks of ROS in mitochondria are both short-term (redox signaling) and long-term (redox regulation of transcription). They influence several different systems: bridge energy metabolism to inflammatory responses via redox-sensitive molecules such as nuclear factor erythroid 2-related factor 2 ( Nrf2) and nuclear factor kappa-light-chain-enhancer of activated $\mathrm{B}$ cells (NFkB); link bioenergetics, autophagy and circadian control; participate in phospholipase activation; associate with oxygen sensing in astrocytes and breathing regulation; and regulate cellular signaling in proliferating cells (Bolanos et al. 2016). A mild increase in ROS generation is associated with the separation of the Nrf2 protein from Keap1 (marker for ubiquitin-dependent proteasomal degradation) and the translocation of $\mathrm{Nrf2}$ to the nucleus and its binding with the antioxidant response element (ARE). The Nrf2-ARE pathway is a very important component of cellular antioxidant, stress adaptation and anti-inflammatory defense systems. Another oxidative stress-responding transcription factor is NFKB, which is activated by hydrogen peroxide. Although mild oxidative stress is increased upon activation of $\mathrm{NFKB}$, higher concentrations of ROS oxidize and inactivate NFKB (Bolisetty and Jaimes 2013, Indo et al. 2017). ROS are markers during fuel sensing and use and also serve as important regulators of hypothalamic neuropeptide- $Y$ and pro-opiomelanocortin neurons to regulate food intake and body weight (Gyengesi et al. 2012). ROS may also activate extracellular endopeptidases referred to as matrix metalloproteinases and influence cell proliferation and differentiation, transcriptional regulation, wound healing, tissue remodeling and other functions (Indo et al. 2017).

\section{Mitochondria as $\mathrm{Ca}^{2+}$ regulators}

Mitochondria play crucial roles in intracellular $\mathrm{Ca}^{2+}$ homeostasis by regulating $\mathrm{Ca}^{2+}$ uptake through the $\mathrm{Ca}^{2+}$ uniporter and $\mathrm{Ca}^{2+}$ efflux via $\mathrm{Na}^{+} / \mathrm{Ca}^{2+}$ exchanger or $\mathrm{mPTP}$, which is very important for synaptic plasticity and for the subsequent regulation of gene transcription $\left(\mathrm{Ca}^{2+}\right.$ ions activate $\mathrm{Ca}^{2+} /$ calmodulin-dependent kinases) (Wang 2007, Srivastava et al. 2018). Mitochondrial $\mathrm{Ca}^{2+}$ also regulates ATP production, mitochondrial metabolism and cell death (Giorgi et al. 2018).

The outer mitochondrial membrane is permeable to $\mathrm{Ca}^{2+}$ through voltage-dependent anion channels, which enable $\mathrm{Ca}^{2+}$ to enter into the IMS. Mitochondria serve as high-capacity transient $\mathrm{Ca}^{2+}$ stores. There is a finite capacity for mitochondrial $\mathrm{Ca}^{2+}$. In high $\mathrm{Ca}^{2+}$ levels, mitochondria maintain low $\mathrm{Ca}^{2+}$ forming calcium phosphate precipitate with inorganic phosphate (Feissner et al. 2009). $\mathrm{Ca}^{2+}$ cross the IMM via the $\mathrm{Ca}^{2+}$ uniporter (a selective $\mathrm{Ca}^{2+}$ channel) when the $\mathrm{Ca}^{2+}$ concentration falls below the submicromolar threshold (rising phase). This transport is possible due to the large negative potential across the IMM. Subsequently $\mathrm{Ca}^{2+}$ is released via a $\mathrm{Na}^{+}$-dependent $\mathrm{Na}^{+} / \mathrm{Ca}^{2+}$ exchanger or $\mathrm{Na}^{+}$-independent $\mathrm{H}^{+} / \mathrm{Ca}^{2+}$ exchanger (both inhibited by $\mathrm{Bcl}-2$ proteins). This $\mathrm{Ca}^{2+}$ cycle is likely linked with the $\mathrm{Ca}^{2+}$ release channels of the endoplasmic reticulum (comprising inositol triphosphate and ryanodine receptors), which serves as a buffer for both intracellular and extracellular $\mathrm{Ca}^{2+}$, enabling mitochondria to participate in $\mathrm{Ca}^{2+}$ signal transduction. Disruptions in mitochondrial membrane potential reduce the ability of mitochondria to maintain $\mathrm{Ca}^{2+}$ homeostasis and result in cell damage. The mPTP connect the outer and inner mitochondrial membrane and is composed of an adenine nucleotide translocator, a voltage-dependent anion channel and the modulatory protein cyclophilin D (Brini 2003, Camello-Almaraz et al. 2006, Wang 2007, D'Orsi et al. 2017). Some studies have suggested that the mPTP is formed from ATP synthase dimers through conformational change, but the specific mechanism of its creation remains unknown (Bernardi et al. 2015). Studies have proposed two gating models of MPTP: one that is irreversible and leads to permanent pore opening and ultimately to cell death and one which the opening is reversible and transient (Hou et al. 2014). Massive mitochondrial $\mathrm{Ca}^{2+}$ concentration might accelerate metabolic enzymes and activate the MPTP opening (resulting in mitochondrial membranes becoming permeable for any molecule smaller than $1.5 \mathrm{kDa}$ ), which permits $\mathrm{Ca}^{2+}$ and proapoptotic molecules, such as apoptosis-inducing factor and cyt $c$ (from complex III), to cross which consequently results in cell death (CamelloAlmaraz et al. 2006, Wang 2007, D'Orsi et al. 2017). Not all mitochondria are equally susceptible to $\mathrm{Ca}^{2+}$-induced mPTP opening. Drahota et al. (2012) proved that cardiac mitochondria of neonatal rats are more resistant to the $\mathrm{Ca}^{2+}$ overload than adult rats. This observation was only confirmed for the cardiac, not the liver mitochondria, probably because of the higher ischemic tolerance of the neonatal heart (Drahota et al. 2012). The mPTP opening is under control of the Bcl-2 family of proteins. This 
family consists of anti-apoptotic proteins such as Bcl-2 and Bcl-xL, which maintain the mPTP in a close state, and pro-apoptotic proteins such as Bax and Bak, which induce mPTP opening and facilitate the transfer of $\mathrm{Ca}^{2+}$ from the ER. The results showed that ROS are able to increase $\mathrm{Ca}^{2+}$ concentration by internal release and disrupt $\mathrm{Ca}^{2+}$-buffering system. It could be said that, in general, mitochondrial inhibitors such as rotenone, myxothiazol or antimycin A decrease $\mathrm{Ca}^{2+}$ oscillation and ROS production. Additionally, any $\mathrm{Ca}^{2+}$ mobilization in mitochondria is a signal for metabolic enzymes to increase productivity, which leads to greater production of ROS; thus, there is a direct connection between the metabolic state and ROS production (Camello-Almaraz et al. 2006, Wang 2007, D'Orsi et al. 2017). Because $\mathrm{Ca}^{2+}$ is part of apoptotic cascade initiation, it is not surprising that many diseases, especially those of a neurodegenerative nature, have been associated with altered $\mathrm{Ca}^{2+}$ homeostasis and abnormal patterns of $\mathrm{Ca}^{2+}$ signals. Disturbances in $\mathrm{Ca}^{2+}$ signaling are very often connected with tissue dysfunction, and it has been proven, that early mitochondrial $\mathrm{Ca}^{2+}$ defects are involved in the pathogenesis of Huntington's disease (Duchen 2000, Brini 2003).

\section{Mitochondrial dysfunction and psychiatric diseases}

Mitochondria might play a crucial role in the pathogenesis of psychiatric disorders, such as depression, anxiety, schizophrenia and bipolar affective disorder (Hroudova and Fisar 2011). The impaired function of OXPHOS and decreased energy production could easily endanger the brain, very dependent on energy supplies. The brain represents around $3 \%$ of body mass but use around $20 \%$ of the oxygen and $25 \%$ of the glucose. Alterations in mitochondrial functions could have the negative impact on the energy consumption of the brain, and increase the vulnerability to psychiatric disorders (Filiou and Sandi 2019).

In brief, wide range of mitochondrial dysfunctions was observed in patients with psychiatric diseases. Polymorphisms and mutations in mitochondrial and nuclear DNA leading to dysregulations of $\mathrm{Ca}^{2+}$, the abnormal cellular energy and metabolism, the decreased $\mathrm{pH}$ and ATP formation, the impaired function of respiratory complexes and enzymes, and increased levels of ROS. The post-mortem examination of patients' brains revealed also anatomical and neuroanatomical abnormalities. More data could be found in the topicrelated literature (Jou et al. 2009, Hroudova and Fisar 2011, Manji et al. 2012, Toker and Agam 2015).

Several studies suggest the possibility that damaged bioenergetics in circulating platelets might project impaired mitochondrial functions in metabolically active organs and help monitor the treatment in patients with Alzheimer's disease or depression. In platelets of depressive patients, the decreased mitochondrial respiration and the drop in maximal capacity of ETC was described. In patients with Alzheimer's disease, the decreased activity of the enzymes of citric acid cycle, the complex III and IV, the increased levels of nitric oxide which form peroxynitrite in reaction with superoxide, and the decreased maximal capacity of ETC was described (Fisar et al. 2019, Petrus et al. 2019). Fisar et al. (2019) observed reduced activity of the citrate synthase and increased activity of the complex I in patients with Alzheimer's disease, and increased activity of the complex II in patients with the Alzheimer's disease and depression.

\section{Effects of psychopharmaca on mitochondrial functions}

Disrupted mitochondrial functions, manifesting as increased oxidative stress, decreased energy production and $\mathrm{Ca}^{2+}$ imbalance have been observed across the whole spectrum of psychiatric disorders (Quiroz et al. 2008, Srivastava et al. 2018). Therefore, how psychopharmaca affect mitochondrial functions is a very interesting phenomenon (Clay et al. 2011). Generally, changes due to mood stabilizers, such as decreased intracellular $\mathrm{pH}$, globally upregulated or downregulated mitochondrial genes, increased expression of the antiapoptotic gene BCL2 and increased $\mathrm{Ca}^{2+}$ storage capacity in the ER have been observed in the brains of patients. Several studies have hypothesized that mood stabilizers induce mitochondrial migration to synaptic terminals, which influences mitochondrial functions and neurotransmitter release and uptake (Scaini et al. 2016). This review is focused on drugs mainly or potentially used in bipolar affective disorder therapy.

\section{Lithium}

Lithium (Li) has protective functions in human cells. It blocks tyrosine phosphorylation of the 2B subtype of the N-methyl-D-aspartate receptor and prevents massive $\mathrm{Ca}^{2+}$ influx and excitotoxicity. Li levels 
in patients with bipolar affective disorder during depressive episodes and complex I activity are positively correlated. Study results also suggest that Li enhances mitochondrial respiration, decreases the proapoptotic enzyme glycogen synthase kinase-3 and inhibits the expression of proapoptotic genes Bax and p53 while simultaneously stimulating antiapoptotic Bcl-2 gene expression (Machado-Vieira et al. 2009, Cikankova et al. 2017). Interesting connection also exists between $\mathrm{Li}, \mathrm{Ca}^{2+}$ and the mPTP. Li is able to augment the mitochondrial $\mathrm{Ca}^{2+}$ capacity, desensitize mitochondria against $\mathrm{Ca}^{2+}$, inhibit calcium-mediated formation of mPTP to suppress membrane depolarization, swelling and the release of cyt $c$ from the mitochondria of rat brain and liver and mitochondria isolated from heart tissue. It also prevents the release of $\mathrm{Ca}^{2+}$ from the ER by blocking the inositol monophosphatase pathway (Shalbuyeva et al. 2007). The same protective effect against $\mathrm{Ca}^{2+}$ overload was also observed in rat brain mitochondria. In homogenates of human prefrontal cortex cells, Li increased the activity of mitochondrial complexes I, II and III. Li also promoted the expression of the scavenger glutathione transferase (Clay et al. 2011). In the rat mania model, Li stimulated the activity of complex I in the prefrontal cortex (Valvassori et al. 2010). In post-mortem brain tissue, Li dose-dependently increases complex I activity (Maurer et al. 2009). Some studies have demonstrated the inhibition of ROS formation and increased glutathione levels after Li application in rat cerebral cells (MachadoVieira et al. 2009). Li also had a cytoprotective effect. $\mathrm{Li}$ treatment inhibited aluminum-induced cyt $c$ translocation, prevented Bcl-2 activity from decreasing and inhibited pro-caspase- 3 cleavage in rabbits (Ghribi et al. 2002). Li also attenuated rotenone-induced proapoptotic caspase-3 activation (King et al. 2001). In another rotenone-Li experiment, Li reduced complex I dysfunction and suppressed ATP production, cellular mortality and apoptosis, and DNA methylation and hydroxymethylation in rat neurons (Scola et al. 2014).

However, not all the articles claim only a positive effect of $\mathrm{Li}$ on mitochondria and cells. $\mathrm{Li}$ lowered complex IV activity in mitochondria isolated from pig brain (Hroudova and Fisar 2010). Because of complex I activity stimulation, it came as no surprise that Li enhanced ROS formation such that mitochondrial structures were damaged. The results showed a decrease in antioxidants and a drop in mitochondrial membrane potential in rat hepatocytes after Li treatment (Eskandari et al. 2012). In mitochondria isolated from rat heart, $\mathrm{Li}$ concentrations from $75-1,000 \mu \mathrm{M}$ inhibited complex II activity and ATP formation, enhanced ROS generation and the activity of proapoptotic caspase-3, caused swelling, and abrogated the mitochondrial membrane potential (Salimi et al. 2017). Similar to the action of VPA, the effect of Li likely depends on many factors.

\section{Mood stabilizing anticonvulsants}

Valproate (VPA) is often associated with the disruption of mitochondrial functions. It has been established, that VPA blocks the entry of long-chain fatty acid into mitochondria (and $\beta$-oxidation) by lowering cofactors such as carnitine and CoA. It also enhances ROS formation, mostly by inhibiting complex II activity and inducing mPTP opening. Higher ROS levels and enhanced MPTP formation lead to a decrease in membrane potential, swelling and release of the cyt $c$ and apoptosis. In rat digitonin-permeabilized hepatocytes, VPA notably inhibited ATP synthesis and pyruvate oxidation when pyruvate was used as a substrate for respiratory reactions. This effect was not observed when succinate or glutamate/malate was used as a substrate gradient (Silva et al. 1997). These results presumed an interaction between VPA and pyruvate, which was confirmed by Aires et al. (2008). VPA and its metabolites inhibited pyruvate uptake in inverted submitochondrial vesicles, which impaired the mitochondria as described above (Aires et al. 2008). Inhibition of 2-oxoglutarate and glutamate-driven OXPHOS has been described in rat liver mitochondria (Luis et al. 2007). Structural abnormalities in the ETC proteins and VPA inhibit complex I and IV activity and decrease superoxide dismutase (Finsterer and Zarrouk Mahjoub 2012, Cikankova et al. 2017, Finsterer and Scorza 2017, Costa et al. 2018). VPA decreased complex I and IV activity in mitochondria isolated from pig brain and facilitated mitochondrial cholesterol transport in Y1 adrenocortical cells (Hroudova and Fisar 2010, Brion et al. 2011).

In contrast, some studies confirmed the inhibition of cyt $c$ release, increased $\mathrm{Bcl}-2$ gene expression and reduced apoptosis via inhibition of the proapoptotic enzyme glycogen synthase kinase-3. Cell protection from glutamate-induced $\mathrm{Ca}^{2+}$ overload and excitotoxicity was also observed (Kazuno et al. 2008, Bachmann et al. 2009, Kato 2011, Cikankova et al. 2017). A similar mitochondria-stabilizing effect was observed in a rat mania model study. Rats pretreated with VPA, in addition to the same results found for LIT, showed lower amphetamine-induced citrate synthase and 
succinate dehydrogenase inhibition (Correa et al. 2007, Feier et al. 2013). VPA also reversed ouabain-induced alterations to the Krebs cycle and mitochondrial enzymes and protected mitochondria from ouabain-induced lipid peroxidation and superoxide formation (Lopes-Borges et al. 2015, Valvassori et al. 2015). Chronic treatment with therapeutic doses of VPA and Li doubled Bcl-2 levels in the frontal cortex of rats (Quiroz et al. 2008). This gene expression was likely mediated via the inhibition of histone deacetylase because deacetylated histones inhibit transcription (Clay et al. 2011). The influence on Bcl-2 seems to be crucial for the beneficial effect of VPA on mitochondria (Bachmann et al. 2009).

The effect of VPA on mitochondrial functions likely depends on several factors, such as the specific type of cell population, presence of mitochondrial pathophysiology, cytotoxic stimulus and, of course, the concentration and duration of VPA treatment (Lai et al. 2006). It seems that VPA confers its cytoprotective effects mostly in the presence of another cytotoxic signal, whereas VPA alone tends to damage mitochondrial functions.

Carbamazepine decreases mitochondrial respiration, ATP production and membrane potential and inhibits the $\mathrm{Ca}^{2+}$-induced swelling of rat liver cells (Cikankova et al. 2017, Finsterer and Scorza 2017).

Lamotrigine effectively inhibits the cytotoxic effect of rotenone and 1-methyl-4-phenypyridinium on PC12 cells, mostly by maintaining the mitochondrial membrane potential, preventing the opening of $\mathrm{MPTP}$ and increasing the levels of glutathione (Kim et al. 2007). The possible neuroprotective effect of lamotrigine may be mediated by respiratory complex I inhibition (Hroudova and Fisar 2011).

\section{Conclusions}

Mitochondria produce ATP supplies using substrates from proteins, lipids and carbohydrate metabolism. In addition to ATP synthesis, mitochondria participate on buffering of free $\mathrm{Ca}^{2+}$ concentration, maintaining of physiological levels of $\mathrm{Ca}^{2+}$, and produce and regulate ROS, trigger intrinsic apoptotic pathway and participate on thermogenesis. These molecules serve as signaling molecules, are involved in the overproduction of ROS or the decreased activity of the antioxidant system damage cellular structure, leading to apoptosis or necrosis and are connected with aging and the pathophysiology of many diseases, including mood disorders.

Altered mitochondrial functions, e.g. suppressed
ATP formation, reduced mitochondrial respiratory complex activity, overproduction of ROS or/and insufficient activity of the antioxidant system, and impaired $\mathrm{Ca}^{2+}$ balance have been observed in affected neurons in people with mood disorders and/or being treated with mood stabilizers. The dysfunctional mitochondrial hypothesis is one of current hypotheses attempts to explain the origin of mood disorders. Many studies have confirmed that mood stabilizers affect mitochondrial functions, even though the exact mechanism or localization of action is unknown. Both inhibition and stimulation of mitochondrial functions was both observed as a result of psychopharmaca treatment. Therefore, changes in mitochondrial parameters induced by mood-stabilizing drugs support the suggestion that mitochondrial dysfunction could be a primary feature of mood disorders. Further knowledge requires the investigation of other psychopharmaca used in bipolar disorder treatment, e.g. antidepressants, benzodiazepines and antipsychotics. The identification of the mechanism(s) of psychopharmaca action, new drug that target mitochondria, and measurable markers of therapy effectiveness is the focus of current biological psychiatry research.

\section{Conflict of Interest}

There is no conflict of interest.

\section{Acknowledgements}

This research was supported by grant No 341/2019 given by the Grant Agency of Charles University, and project No SVV 260373 of Charles University, Czech Republic.

\section{Abbreviations}

ARE - antioxidant response element, ATP - adenosine triphosphate, CoA - coenzyme A, CoQ - oxidized coenzyme Q10, $\mathrm{CoQH}_{2}$ - reduced coenzyme Q10, cyt $c$ cytochrome $c$, ETC - electron transport chain, $\mathrm{FAD}^{+}-$ oxidized form of flavin adenine dinucleotide, $\mathrm{FADH}_{2}-$ reduced form of flavin adenine dinucleotide, IMM - inner mitochondrial membrane, IMS - intermembrane space, $\mathrm{Li}$ - lithium, $\mathrm{mPTP}$ - mitochondrial permeability transition pore, $\mathrm{NAD}^{+}$- oxidized form of nicotinamide adenine dinucleotide, NADH - reduced form of nicotinamide adenine dinucleotide, $\mathrm{NF \kappa B}$ - nuclear factor kappa-lightchain-enhancer of activated B cells, Nrf2 - nuclear factor erythroid 2-related factor 2, OXPHOS - oxidative phosphorylation, PMF - proton motive force, ROS reactive oxygen species, VPA - valproate. 


\section{References}

AIRES CC, SOVERAL G, LUIS PB, TEN BRINK HJ, DE ALMEIDA IT, DURAN M, WANDERS RJ, SILVA MF: Pyruvate uptake is inhibited by valproic acid and metabolites in mitochondrial membranes. FEBS Lett 582: 3359-3366, 2008.

ANDREAZZA AC, NIERENBERG AA: Mitochondrial dysfunction: at the core of psychiatric disorders? Biol Psychiatry 83: 718-719, 2018.

ANGELOVA PR, ABRAMOV AY: Role of mitochondrial ROS in the brain: from physiology to neurodegeneration. FEBS Lett 592: 692-702, 2018.

BACHMANN RF, WANG Y, YUAN P, ZHOU R, LI X, ALESCI S, DU J, MANJI HK: Common effects of lithium and valproate on mitochondrial functions: protection against methamphetamine-induced mitochondrial damage. Int J Neuropsychopharmacol 12: 805-822, 2009.

BARCA E, GANETZKY RD, POTLURI P, JUANOLA-FALGARONA M, GAI X, LI D, JALAS C, HIRSCH Y, EMMANUELE V, TADESSE S, ZIOSI M, AKMAN HO, CHUNG WK, TANJI K, MCCORMICK EM, PLACE E, CONSUGAR M, PIERCE EA, HAKONARSON H, WALLACE DC, HIRANO M, FALK MJ: USMG5 Ashkenazi Jewish founder mutation impairs mitochondrial complex V dimerization and ATP synthesis. Hum Mol Genet 27: 3305-3312, 2018.

BERNARDI P, DI LISA F, FOGOLARI F, LIPPE G: From ATP to PTP and back: a dual function for the mitochondrial ATP synthase. Circ Res 116: 1850-1862, 2015.

BERTERO E, MAACK C: Calcium signaling and reactive oxygen species in mitochondria. Circ Res 122: 1460-1478, 2018.

BOLANOS JP, CADENAS E, DUCHEN MR, HAMPTON MB, MANN GE, MURPHY MP: Introduction to special issue on mitochondrial redox signaling in health and disease. Free Radic Biol Med 100: 1-4, 2016.

BOLISETTY S, JAIMES EA: Mitochondria and reactive oxygen species: physiology and pathophysiology. Int J Mol Sci 14: 6306-6344, 2013.

BOUCHEZ C, DEVIN A: Mitochondrial biogenesis and mitochondrial reactive oxygen species (ROS): a complex relationship regulated by the cAMP/PKA signaling pathway. Cells 8: E287, 2019.

BRINI M: Ca2+ signalling in mitochondria: mechanism and role in physiology and pathology. Cell Calcium 34: 399-405, 2003.

BRION L, GOROSTIZAGA A, GOMEZ NV, PODESTA EJ, CORNEJO MACIEL F, PAZ C: Valproic acid alters mitochondrial cholesterol transport in Y1 adrenocortical cells. Toxicol In Vitro 25: 7-12, 2011.

CAMELLO-ALMARAZ C, GOMEZ-PINILLA PJ, POZO MJ, CAMELLO PJ: Mitochondrial reactive oxygen species and Ca2+ signaling. Am J Physiol Cell Physiol 291: C1082-C1088, 2006.

CECCHINI G: Respiratory complex II: role in cellular physiology and disease. Biochim Biophys Acta 1827: 541-542, 2013.

CEDIKOVA M, PITULE P, KRIPNEROVA M, MARKOVA M, KUNCOVA J: Multiple roles of mitochondria in aging processes. Physiol Res 65 (Suppl 5): S519-S531, 2016.

CHANDEL NS, MALTEPE E, GOLDWASSER E, MATHIEU CE, SIMON MC, SCHUMACKER PT: Mitochondrial reactive oxygen species trigger hypoxia-induced transcription. Proc Natl Acad Sci U S A 95: 11715-11720, 1998.

CIKANKOVA T, SIGITOVA E, ZVEROVA M, FISAR Z, RABOCH J, HROUDOVA J: Mitochondrial dysfunctions in bipolar disorder: effect of the disease and pharmacotherapy. CNS Neurol Disord Drug Targets 16: 176-186, 2017.

CLAY HB, SILLIVAN S, KONRADI C: Mitochondrial dysfunction and pathology in bipolar disorder and schizophrenia. Int J Dev Neurosci 29: 311-324, 2011.

CORREA C, AMBONI G, ASSIS LC, MARTINS MR, KAPCZINSKI F, STRECK EL, QUEVEDO J: Effects of lithium and valproate on hippocampus citrate synthase activity in an animal model of mania. Prog Neuropsychopharmacol Biol Psychiatry 31: 887-891, 2007.

COSTA RMD, KARMIRIAN K, REHEN SK: Deformation of mitochondrial cristae in human neural progenitor cells exposed to valproic acid. An Acad Bras Cienc 90: 2223-2232, 2018. 
D'ORSI B, MATEYKA J, PREHN JHM: Control of mitochondrial physiology and cell death by the Bcl-2 family proteins Bax and Bok. Neurochem Int 109: 162-170, 2017.

DAIBER A, DI LISA F, OELZE M, KROLLER-SCHON S, STEVEN S, SCHULZ E, MUNZEL T: Crosstalk of mitochondria with NADPH oxidase via reactive oxygen and nitrogen species signalling and its role for vascular function. Br J Pharmacol 174: 1670-1689, 2017.

DE GIUSTI VC, CALDIZ CI, ENNIS IL, PEREZ NG, CINGOLANI HE, AIELLO EA: Mitochondrial reactive oxygen species (ROS) as signaling molecules of intracellular pathways triggered by the cardiac renin-angiotensin IIaldosterone system (RAAS). Front Physiol 4: 126, 2013.

DE SOUSA RT, MACHADO-VIEIRA R, ZARATE CA JR, MANJI HK: Targeting mitochondrially mediated plasticity to develop improved therapeutics for bipolar disorder. Expert Opin Ther Targets 18: 1131-1147, 2014.

DIVAKARUNI AS, BRAND MD: The regulation and physiology of mitochondrial proton leak. Physiology (Bethesda) 26: 192-205, 2011.

DRAHOTA Z, MILEROVA M, ENDLICHER R, RYCHTRMOC D, CERVINKOVA Z, OSTADAL B: Developmental changes of the sensitivity of cardiac and liver mitochondrial permeability transition pore to calcium load and oxidative stress. Physiol Res 61 (Suppl 1): S165-S172, 2012.

DUBOUCHAUD H, WALTER L, RIGOULET M, BATANDIER C: Mitochondrial NADH redox potential impacts the reactive oxygen species production of reverse Electron transfer through complex I. J Bioenerg Biomembr 50: 367-377, 2018.

DUCHEN MR: Mitochondria and Ca(2+)in cell physiology and pathophysiology. Cell Calcium 28: 339-348, 2000.

ESKANDARI MR, FARD JK, HOSSEINI MJ, POURAHMAD J: Glutathione mediated reductive activation and mitochondrial dysfunction play key roles in lithium induced oxidative stress and cytotoxicity in liver. Biometals 25: 863-873, 2012.

FEIER G, VALVASSORI SS, VARELA RB, RESENDE WR, BAVARESCO DV, MORAIS MO, SCAINI G, ANDERSEN ML, STRECK EL, QUEVEDO J: Lithium and valproate modulate energy metabolism in an animal model of mania induced by methamphetamine. Pharmacol Biochem Behav 103: 589-596, 2013.

FEISSNER RF, SKALSKA J, GAUM WE, SHEU SS: Crosstalk signaling between mitochondrial Ca2+ and ROS. Front Biosci (Landmark Ed) 14: 1197-1218, 2009.

FILIOU MD, SANDI C: Anxiety and brain mitochondria: a bidirectional crosstalk. Trends Neurosci 42: 573-588, 2019.

FINSTERER J, SCORZA FA: Effects of antiepileptic drugs on mitochondrial functions, morphology, kinetics, biogenesis, and survival. Epilepsy Res 136: 5-11, 2017.

FINSTERER J, ZARROUK MAHJOUB S: Mitochondrial toxicity of antiepileptic drugs and their tolerability in mitochondrial disorders. Expert Opin Drug Metab Toxicol 8: 71-79, 2012.

FISAR Z, HANSIKOVA H, KRIZOVA J, JIRAK R, KITZLEROVA E, ZVEROVA M, HROUDOVA J, WENCHICH L, ZEMAN J, RABOCH J: Activities of mitochondrial respiratory chain complexes in platelets of patients with Alzheimer's disease and depressive disorder. Mitochondrion 48: 66-77, 2019.

GHRIBI O, HERMAN MM, SPAULDING NK, SAVORY J: Lithium inhibits aluminum-induced apoptosis in rabbit hippocampus, by preventing cytochrome $\mathrm{c}$ translocation, Bcl-2 decrease, Bax elevation and caspase-3 activation. J Neurochem 82: 137-145, 2002.

GIORGI C, MARCHI S, PINTON P: The machineries, regulation and cellular functions of mitochondrial calcium. Nat Rev Mol Cell Biol 19: 713-730, 2018.

GRIMM S: Respiratory chain complex II as general sensor for apoptosis. Biochim Biophys Acta 1827: 565-572, 2013.

GYENGESI E, PAXINOS G, ANDREWS ZB: Oxidative stress in the hypothalamus: the importance of calcium signaling and mitochondrial ROS in body weight regulation. CurrNeuropharmacol 10: 344-353, 2012.

HIRST J: Towards the molecular mechanism of respiratory complex I. Biochem J 425: 327-339, 2009.

HOU T, WANG X, MA Q, CHENG H: Mitochondrial flashes: new insights into mitochondrial ROS signalling and beyond. J Physiol 592: 3703-3713, 2014.

HROUDOVA J, FISAR Z: Activities of respiratory chain complexes and citrate synthase influenced by pharmacologically different antidepressants and mood stabilizers. Neuro Endocrinol Lett 31: 336-342, 2010.

HROUDOVA J, FISAR Z: Connectivity between mitochondrial functions and psychiatric disorders. Psychiatry Clin Neurosci 65: 130-141, 2011. 
HROUDOVÁ J, FIŠAR Z: Control mechanism in mitochondrial oxidative phosphorylation. Neural Regen Res 8: 13, 2013.

INDO HP, HAWKINS CL, NAKANISHI I, MATSUMOTO KI, MATSUI H, SUENAGA S, DAVIES MJ, ST CLAIR DK, OZAWA T, MAJIMA HJ: Role of mitochondrial reactive oxygen species in the activation of cellular signals, molecules, and function. Handb Exp Pharmacol 240: 439-456, 2017.

IVERSON TM: Catalytic mechanisms of complex II enzymes: a structural perspective. Biochim Biophys Acta 1827: 648-657, 2013.

IWATA S, LEE JW, OKADA K, LEE JK, IWATA M, RASMUSSEN B, LINK TA, RAMASWAMY S, JAP BK: Complete structure of the 11-subunit bovine mitochondrial cytochrome bc1 complex. Science 281: 64-71, 1998.

JASTROCH M, DIVAKARUNI AS, MOOKERJEE S, TREBERG JR, BRAND MD: Mitochondrial proton and electron leaks. Essays Biochem 47: 53-67, 2010.

JONCKHEERE AI, HUIGSLOOT M, LAMMENS M, JANSEN J, VAN DEN HEUVEL LP, SPIEKERKOETTER U, VON KLEIST-RETZOW JC, FORKINK M, KOOPMAN WJ, SZKLARCZYK R, HUYNEN MA, FRANSEN JA, SMEITINK JA, RODENBURG RJ: Restoration of complex V deficiency caused by a novel deletion in the human TMEM70 gene normalizes mitochondrial morphology. Mitochondrion 11: 954-963, 2011.

JONCKHEERE AI, SMEITINK JA, RODENBURG RJ: Mitochondrial ATP synthase: architecture, function and pathology. J Inherit Metab Dis 35: 211-225, 2012.

JOU SH, CHIU NY, LIU CS: Mitochondrial dysfunction and psychiatric disorders. Chang Gung Med J 32: 370-379, 2009.

KATO T: Mitochondrial dysfunction and bipolar disorder. Curr Top Behav Neurosci 5: 187-200, 2011.

KAZUNO AA, MUNAKATA K, KATO N, KATO T: Mitochondrial DNA-dependent effects of valproate on mitochondrial calcium levels in transmitochondrial cybrids. Int J Neuropsychopharmacol 11: 71-78, 2008.

KIM YJ, KO HH, HAN ES, LEE CS: Lamotrigine inhibition of rotenone- or 1-methyl-4-phenylpyridinium-induced mitochondrial damage and cell death. Brain Res Bull 71: 633-640, 2007.

KING TD, BIJUR GN, JOPE RS: Caspase-3 activation induced by inhibition of mitochondrial complex I is facilitated by glycogen synthase kinase-3beta and attenuated by lithium. Brain Res 919: 106-114, 2001.

LAI JS, ZHAO C, WARSH JJ, LI PP: Cytoprotection by lithium and valproate varies between cell types and cellular stresses. Eur J Pharmacol 539: 18-26, 2006.

LANCIANO P, KHALFAOUI-HASSANI B, SELAMOGLU N, GHELLI A, RUGOLO M, DALDAL F: Molecular mechanisms of superoxide production by complex III: a bacterial versus human mitochondrial comparative case study. Biochim Biophys Acta 1827: 1332-1339, 2013.

LENAZ G: Mitochondria and reactive oxygen species. Which role in physiology and pathology? Adv Exp Med Biol 942: 93-136, 2012.

LIU J, FU X, CHANG Z: A reciprocating motion-driven rotation mechanism for the ATP synthase. Sci China Life Sci 59: 44-48, 2016.

LODISH H, BERK A, KAISER CA, KRIEGER M, BRETSCHER A, PLOEGH H, AMON A, SCOTT MP: Molecular Cell Biology. WH FREEMAN (ed.), New York, 2013.

LOPES-BORGES J, VALVASSORI SS, VARELA RB, TONIN PT, VIEIRA JS, GONCALVES CL, STRECK EL, QUEVEDO J: Histone deacetylase inhibitors reverse manic-like behaviors and protect the rat brain from energetic metabolic alterations induced by ouabain. Pharmacol Biochem Behav 128: 89-95, 2015.

LUIS PB, RUITER JP, AIRES CC, SOVERAL G, DE ALMEIDA IT, DURAN M, WANDERS RJ, SILVA MF: Valproic acid metabolites inhibit dihydrolipoyl dehydrogenase activity leading to impaired 2-oxoglutaratedriven oxidative phosphorylation. Biochim Biophys Acta 1767: 1126-1133, 2007.

MACHADO-VIEIRA R, MANJI HK, ZARATE CA JR: The role of lithium in the treatment of bipolar disorder: convergent evidence for neurotrophic effects as a unifying hypothesis. Bipolar Disord 11 (Suppl 2): 92-109, 2009.

MAILLOUX RJ, HARPER ME: Mitochondrial proticity and ROS signaling: lessons from the uncoupling proteins. Trends Endocrinol Metab 23: 451-458, 2012. 
MANJI H, KATO T, DI PROSPERO NA, NESS S, BEAL MF, KRAMS M, CHEN G: Impaired mitochondrial function in psychiatric disorders. Nat Rev Neurosci 13: 293-307, 2012.

MAURER IC, SCHIPPEL P, VOLZ HP: Lithium-induced enhancement of mitochondrial oxidative phosphorylation in human brain tissue. Bipolar Disord 11: 515-522, 2009.

NESCI S, TROMBETTI F, VENTRELLA V, PAGLIARANI A: The c-ring of the F1FO-ATP synthase: facts and perspectives. J Membr Biol 249: 11-21, 2016.

NEUPANE P, BHUJU S, THAPA N, BHATTARAI HK: ATP synthase: structure, function and inhibition. Biomol Concepts 10: 1-10, 2019.

OHNISHI T, OHNISHI ST, SALERNO JC: Five decades of research on mitochondrial NADH-quinone oxidoreductase (complex I). Biol Chem 399: 1249-1264, 2018.

PETRUS AT, LIGHEZAN DL, DANILA MD, DUICU OM, STURZA A, MUNTEAN DM, IONITA I: Assessment of platelet respiration as emerging biomarker of disease. Physiol Res 68: 347-363, 2019.

QUIROZ JA, GRAY NA, KATO T, MANJI HK: Mitochondrially mediated plasticity in the pathophysiology and treatment of bipolar disorder. Neuropsychopharmacology 33: 2551-2565, 2008.

RODRIGUEZ-HERNANDEZ A, CORDERO MD, SALVIATI L, ARTUCH R, PINEDA M, BRIONES P, GOMEZ IZQUIERDO L, COTAN D, NAVAS P, SANCHEZ-ALCAZAR JA: Coenzyme Q deficiency triggers mitochondria degradation by mitophagy. Autophagy 5: 19-32, 2009.

SALIMI A, GHOLAMIFAR E, NASERZADEH P, HOSSEINI MJ, POURAHMAD J: Toxicity of lithium on isolated heart mitochondria and cardiomyocyte: a justification for its cardiotoxic adverse effect. J Biochem Mol Toxicol 31: 2017. doi:10.1002/jbt.21836

SCAINI G, REZIN GT, CARVALHO AF, STRECK EL, BERK M, QUEVEDO J: Mitochondrial dysfunction in bipolar disorder: evidence, pathophysiology and translational implications. Neurosci Biobehav Rev 68: 694-713, 2016.

SCOLA G, KIM HK, YOUNG LT, SALVADOR M, ANDREAZZA AC: Lithium reduces the effects of rotenoneinduced complex I dysfunction on DNA methylation and hydroxymethylation in rat cortical primary neurons. Psychopharmacology (Berl) 231: 4189-4198, 2014.

SHALBUYEVA N, BRUSTOVETSKY T, BRUSTOVETSKY N: Lithium desensitizes brain mitochondria to calcium, antagonizes permeability transition, and diminishes cytochrome C release. J Biol Chem 282: 18057-18068, 2007.

SILVA MF, RUITER JP, ILLST L, JAKOBS C, DURAN M, DE ALMEIDA IT, WANDERS RJ: Valproate inhibits the mitochondrial pyruvate-driven oxidative phosphorylation in vitro. J Inherit Metab Dis 20: 397-400, 1997.

SPEIJER D: Can all major ROS forming sites of the respiratory chain be activated by high $\mathrm{FADH}_{2} / \mathrm{NADH}$ ratios? Ancient evolutionary constraints determine mitochondrial ROS formation. Bioessays 41: e1800180, 2018.

SRIVASTAVA R, FAUST T, RAMOS A, ISHIZUKA K, SAWA A: Dynamic changes of the mitochondria in psychiatric illnesses: new mechanistic insights from human neuronal models. Biol Psychiatry 83: 751-760, 2018.

TOKER L, AGAM G: Mitochondrial dysfunction in psychiatric morbidity: current evidence and therapeutic prospects. Neuropsychiatr Dis Treat 11: 2441-2447, 2015.

VALVASSORI SS, RESENDE WR, LOPES-BORGES J, MARIOT E, DAL-PONT GC, VITTO MF, LUZ G, DE SOUZA CT, QUEVEDO J: Effects of mood stabilizers on oxidative stress-induced cell death signaling pathways in the brains of rats subjected to the ouabain-induced animal model of mania: mood stabilizers exert protective effects against ouabain-induced activation of the cell death pathway. J Psychiatr Res 65: 63-70, 2015.

VALVASSORI SS, REZIN GT, FERREIRA CL, MORETTI M, GONCALVES CL, CARDOSO MR, STRECK EL, KAPCZINSKI F, QUEVEDO J: Effects of mood stabilizers on mitochondrial respiratory chain activity in brain of rats treated with d-amphetamine. J Psychiatr Res 44: 903-909, 2010.

WANG JF: Defects of mitochondrial electron transport chain in bipolar disorder: implications for mood-stabilizing treatment. Can J Psychiatry 52: 753-762, 2007. 